\title{
Verborgene Datenschätze? (Wirkungsorientierte) Evaluierung auf Basis prozessproduzierter Daten in sozialen Einrichtungen
}

\author{
Peter Stepanek, ${ }^{1}$ Florentina Astleithner, ${ }^{1}$ Stefanie Reiter, ${ }^{2}$ \\ Olaf Lobermeier, ${ }^{3}$ Frank Buchheit ${ }^{4}$
}

\section{Schwerpunkt Wirkungsorientierung in sozialwirtschaftlichen Organisationen: Rahmung der Frühjahrstagung 2019 des AK Soziale Dienstleistungen der DeGEval}

Aufgrund der zunehmenden Digitalisierung und einer wachsenden Fokussierung von Fördergeber(inne)n auf Wirkungsorientierung müssen sich auch soziale Einrichtungen verstärkt mit den eigenen Daten beschäftigen. Ein genauer Blick auf diese Daten kann neue Perspektiven eröffnen, wenn man bspw. deren Potenzial für die Evaluierung wahrnimmt. Denn nicht immer müssen Daten anlassbezogen erhoben werden. Soziale Einrichtungen sitzen auf einem wahren ,Schatz' von prozessproduzierten Daten (z.B. Schriftstücke, Zahlen und Statistiken). Diese entstehen auf vielfältige Art und Weise in der Arbeit mit Klient(inn)en, in internen Organisationsprozessen sowie in der Kommunikation mit externen Stakeholdern (wie z.B. Fördergeber(innen), Spender(innen)). Dabei können vorhandene Datendokumentationen mehr als die Darstellung des Outputs in Jahresberichten oder Statistiken für die Fördergeber(innen) erlauben. Sie können durchaus wichtige Informationen zur Wirkung und zum Nutzen der Angebote liefern und somit eine gute Basis für die Erhebung zusätzlicher Informationen darstellen oder eine solche unnötig machen. Das wird zu einer Win-Win-Situation für die Einrichtungen: Die Dokumentation kann aus einer anderen Nutzenperspektive betrachtet und ein wirkungsorientiertes Monitoring gefördert werden. Das Zugreifen auf bestehende Daten kann zudem

FH Campus Wien, Sozialwirtschaft und Soziale Arbeit

Deutsches Jugendinstitut e.V., Außenstelle Halle (Saale)

proval Gesellschaft für sozialwissenschaftliche Analyse - Beratung - Evaluation, Hannover

4 Landeskriminalamt Baden-Württemberg, Stuttgart 
den Aufwand bei der Konzeption der Evaluierung oder des Monitorings reduzieren. Trotz der Vorteile ist die Arbeit mit prozessproduzierten Daten auch aus einer kritischen Perspektive zu betrachten. So müssen neben dem Datenschutz ethische Überlegungen miteinbezogen werden, wenn personenbezogene und sensible Daten weiter ausgewertet werden. Als Herausforderung kann sich bspw. auch der unterschiedliche Dokumentationsstil verschiedener Mitarbeiter(innen) sozialer Einrichtungen oder Professionen entpuppen. Aus einer Auseinandersetzung mit dem Thema wird deutlich, wie wichtig die Berücksichtigung bereits vorhandener Daten und deren Erhebung für eine kontinuierliche und produktive Implementierung von Wirkungsdarstellungen ist und dass man die Evaluierung bereits bei der Konzeption der Dokumentation neuer Angebote mitdenken kann.

Die Frühjahrstagung 2019 des Arbeitskreises Soziale Dienstleistungen in der Gesellschaft für Evaluation (DeGEval) ${ }^{5}$ „Verborgene Datenschätze? (Wirkungsorientierte) Evaluierung auf Basis prozessproduzierter Daten in sozialen Einrichtungen" beleuchtete die Evaluierung anhand prozessproduzierter Daten aus verschiedenen Perspektiven. Sie führte vor diesem Hintergrund im Handlungsfeld tätige Vertreter(innen) sozialer Einrichtungen, von Forschungseinrichtungen und von Hochschulen, von Fördergeber(inne)n und aus der Evaluationspraxis zusammen. Die internationale Tagung, zu der vom Europäischen Masterstudium Sozialwirtschaft und Soziale Arbeit der FH Campus Wien eingeladen wurde, fand am 28. und 29. Mai 2019 statt. Rund 60 Personen aus Österreich, Deutschland und Luxemburg nahmen daran teil. Das Thema wurde dabei von Florentina Astleithner und Peter Stepanek aus dem Studiengang eingebracht. Es leitete sich aus ihrer langjährigen Auseinandersetzung mit Fragen der Wirkungsorientierung in sozialwirtschaftlichen Organisationen ab. Das Ziel des Erfahrungsaustauschs war, unterschiedliche Perspektiven zusammenzuführen und Handlungslogiken aufzuzeigen sowie gemeinsam Herausforderungen, Ansätze und methodische Zugänge feldübergreifend zu diskutieren.

Bereits im Vorfeld der Tagung war ein großes Interesse an diesem Thema und den damit aufgeworfenen Fragen spürbar, das sich in der Anzahl der Anmeldungen niederschlug. Der erste Tag umfasste vier Beiträge von Referierenden aus Österreich und Deutschland, die aus diversen Bereichen des Sozialen und Blickwinkeln der Fachpraxis sowie der internen und externen Evaluation Herangehensweisen und Ansätze schilderten und die damit verbundenen Grenzen und Potenziale reflektierten. ${ }^{6}$ Im Folgenden werden die Inhalte des ersten Tages zusammengefasst.

5 Der Arbeitskreis Soziale Dienstleistungen der Gesellschaft für Evaluation bietet ein Austauschforum für Personen, die im sozialen Bereich mit dem Thema Evaluation befasst sind. Der AK greift dabei methodische Fragen der Evaluation im Handlungsfeld auf, das durch spezifische Leistungscharakteristika und über spezielle Zugänge zu Fragen der Evaluation gekennzeichnet ist. Gegenstand der Diskussion und Ziel ist die Reflexion der Evaluationspraxis und deren methodische und inhaltliche Weiterentwicklung (vgl. www.degeval.org/de/arbeits kreise/soziale-dienstleistungen/ [12.01.2020]).

6 Das Programm der Frühjahrstagung ist unter https://www.degeval.org/arbeitskreise/soziale-dienst leistungen/ [12.01.2020] abrufbar. 


\section{Vier Keynotes aus Österreich und Deutschland}

Am ersten Tagungstag widmete Diana Zierold vom Deutschen Jugendinstitut ihre praxisorientierte Keynote aus Sicht einer ehemaligen Einrichtungsleiterin den Möglichkeiten der Zusammenführung verschiedener Daten in Wohnstätten für Menschen mit Behinderungen und pflegebedürftige Menschen zum Wohl der Bewohner(innen). Dabei ging sie der leitenden Frage nach, inwieweit die Standards der DeGEval ${ }^{7}$ ein gutes Instrumentarium für die Durchführung von internen Evaluationen in sozialen Einrichtungen darstellen und dabei helfen, die Qualität in der Pflege und Betreuung zu verbessern. Sie stellte verschiedene Instrumente zur Dokumentation vor (H.M.B.W.-Verfahren-Bogen, Demenzscreening, Verhaltensbogen) und beleuchtete, wie die Daten aus diesen Tools in die Konzeption neuer Angebote einfließen können. Bei der Evaluierung auf Basis dieser Daten gelte es viele Herausforderungen zu überwinden. Es brauche Zeit, entsprechende fachliche Expertise, Know-how in der Anwendung und das Commitment des Personals der Einrichtung.

Frank Bauer und Jan Gellermann vom Institut für Arbeitsmarkt- und Berufsforschung der Bundesagentur für Arbeit (IAB) ließen die Teilnehmenden an ihren Erfahrungen bei der kombinierten Nutzung von Prozessdaten, Befragungen und Hilfedokumentationen teilhaben. Im Zentrum standen verschiedene externe Evaluationen von Arbeitsmarktprogrammen für Langzeitarbeitslose. Mit großen Datenmengen, die verknüpft wurden, konnten Aussagen zur Wirkung verschiedener Maßnahmen getroffen werden, wobei auch Kontrollgruppendesigns auf Basis statistischer Matching-Verfahren zum Einsatz kamen. Es wurden von den Evaluierenden Mixed-Methods-Designs entwickelt, um zu verstehen, was in den Maßnahmen tatsächlich passiert und wie die unterschiedlichen Unterstützungsformen wirken. Auch statistische Modelle, die Aussagen über Netto-Effekte in Bezug auf die Integration in den ersten Arbeitsmarkt ermöglichten, seien dabei ergänzungsbedürftig, wenn man Genaueres über die Inhalte der Maßnahmen und deren Einfluss erfahren möchte. Hierzu bedarf es Wissens über die Erfahrungen und Einschätzungen der Maßnahmeteilnehmenden (individuelle Probleme, Verhältnis zu Ausbilder(in), Alltagsorganisation, Lernanforderungen, etc.) und der umsetzenden Fachkräfte (Implementation, Programmfehler, Art und Inhalt der Sozialarbeit). Diese Informationen könne man bspw. durch ergänzende Befragungen und Auswertung von Hilfedokumentationen gewinnen, sofern diese prozessbegleitend angefertigt werden und für die Evaluation zur Verfügung stehen. Herausfordernd stellten sich in diesem Kontext mitunter die unterschiedlichen Interessen der umsetzenden Träger sowie fehlende Richtlinien dar.

Markus Schwab und Elisabeth Stefanek erläuterten die Nutzung von prozessproduzierten Daten für die Evaluation und Qualitätsentwicklung in der pro-menteGruppe Kärnten. Sie wiesen einleitend darauf hin, dass Qualitätssicherung und -entwicklung auch im Gesundheitswesen zunehmend an Bedeutung gewinne. Die interne Forschungsabteilung hat dafür ein umfassendes Datenmanagementsystem partizipativ mit Mitarbeitenden entwickelt. Abgebildet sind darin alle Daten, die im

7 Verfügbar unter: https://www.degeval.org/degeval-standards/standards-fuer-evaluation/ [12.01.2020]. 
Laufe des Betreuungsprozesses (vom Erstkontakt bis hin zum Fallabschluss) gesammelt werden. Diese können für diverse Fragestellungen im Rahmen der kontinuierlichen Evaluation und für die Qualitätssicherung ausgewertet und für verschiedene User(innen)gruppen aufbereitet werden. So stehe ein wahrer Datenschatz für Fördergerber(innen), den internen Gebrauch im Sinne der Verbesserung und für die interne und externe Evaluierung zur Verfügung. Trotz Einschränkungen und methodischer Herausforderungen (bspw. Umgang mit fehlenden Werten, nicht systematisch geplanten Erhebungszeiträumen) sehen sie einen Nutzen für die Forschung und Qualitätsentwicklung, bei der Erprobung und Weiterentwicklung von Methoden, bei der Abstimmung interner und externer Informationsbedürfnisse, bei der Etablierung einer Evaluationskultur auf Organisationsebene sowie beim Ausbau der Evaluationskompetenz und als Motivationsfaktor auf Seiten der Mitarbeitenden.

Katrin Schöber und Bernhard Rubik von der Wiener Wohnen Kundenservice GmbH beleuchteten die Verknüpfung von Dokumentation und Evaluierung im Case Management aus Sicht einer internen Datenanalystin und eines Case Managers. Das Case Management ist dabei ein Service für Mieter(innen), die sich in schwierigen Lebenssituationen befinden. Sie betonten den Stellenwert einer internen Datenanalystin, die Hand in Hand mit dem Team der Sozialarbeit die Dokumentation und die Datenanalyse weiterentwickelt. Die Referierenden hatten zahlreiche Praxistipps für die Teilnehmenden wie die Dokumentation und die Evaluation konzeptionell von Beginn an zusammenzudenken, den Aufwand für die Dokumentation auBerhalb der Fallbeschreibung eher gering zu halten und ein generelles Verständnis für den Nutzen von Dokumentation und Datenmanagement im Team zu schaffen. Lezteres kann bspw. durch Ergebnispräsentationen und gemeinsame Interpretationen im Team sowie die partizipative Entwicklung von Standards für die Dokumentation erreicht werden. Dokumentationsstandards sollten verständlich und nachvollziehbar sowie angemessen sein und zwar in Hinblick auf unterschiedliche Verwertungsinteressen und Handlungslogiken. Durch dieses Vorgehen können verborgene Datenschätze sichtbar und nutzbar gemacht werden.

Am Schluss hielten Stefanie Reiter und Frank Buchheit vom AK Soziale Dienstleistungen der DeGEval wesentliche Erkenntnisse und Diskussionspunkte des ersten Veranstaltungstages fest: Es wurde eine Vielfalt von Perspektiven aus Forschung und Praxis beleuchtet. Aus den Beiträgen aus unterschiedlichen Bereichen des Sozialen wurde ersichtlich, dass diese nicht nur Gemeinsamkeiten in der Arbeit mit teilweise schwer erreichbaren Zielgruppen, sondern auch Herausforderungen in der Vereinbarung von unterschiedlichen Erwartungen und Handlungslogiken auf Seiten der Stakeholer (insb. Evaluierende, umsetzende Fachpraxis, Klient(inn)en, Fördergeber(innen)) aufweisen. Diese gelte es zu reflektieren. Der Fokus und der Nutzen der Evaluierung anhand der prozessproduzierten Daten müsse der Einrichtung jeweils klar sein. In vielen Fällen müssten diese vielfältig vorhandenen Daten aber zunächst aufbereitet werden, um sie für verschiedene User(innen)gruppen nutzbar zu machen. Gerade die Variation an Datenquellen und das systematische Zusammenführen mit verschiedenen Auswertungsmethoden könnten als Chance gesehen werden. Das verlange nach dem Ausbau einer Evaluationskultur und nach evaluati- 
vem Denken im Team. Generell können Evaluierungen helfen, Prozesse, Leistungen und Effekte aufzuzeigen, noch bevor es verlangt werde.

\section{Drei praxisnahe Workshops}

Am zweiten Tag standen drei Workshops für die Teilnehmenden zur Auswahl. Dagmar Holzer (Streetwork Oberösterreich) und Florentina Astleithner (FH Campus Wien, Sozialwirtschaft und Soziale Arbeit) widmeten sich am Beispiel Streetwork der Nutzung prozessproduzierter Daten zur Wirkungsdarstellung. Der Workshop von Isabella Hager und Christine Reidl (prospect $\mathrm{GmbH}$ ) zeigte am Beispiel begleitende Evaluation des Stufenmodells der Integration in Niederösterreich, dass es nützlich ist, die Dokumentation von Beginn an mit Blick auf deren Auswertung aufzubauen, um systematisch daraus lernen zu können. Bei Wolfgang Haydn und Martina Burtscher (Oasis Socialis Transition) standen die Erfahrungen aus der Nutzung prozessproduzierter Daten zur Evaluierung der Arbeit mit psychisch erkrankten Personen anhand der von ihnen entwickelten Tools im Mittelpunkt.

\section{Fazit und Ausblick}

Für Peter Stepanek (FH Campus Wien, Sozialwirtschaft und Soziale Arbeit) der für die Organisation und die Moderation der Konferenz verantwortlich war, hat sich einmal mehr gezeigt, wie sinnvoll es ist, die Themen Wirkung, Evaluation und Dokumentation gemeinsam zu denken. Es gäbe ein großes Potenzial, Ressourcen zu sparen, aber vor allem neue Erkenntnisse zu gewinnen. Die voranschreitende Digitalisierung der Dokumentation Sozialer Arbeit werde mehr Potenzial zur Nutzung bieten, aber auch nach speziellem Know-how verlangen. Das Thema Datenmanagement als Grundlage für eine wirkungsorientierte Evaluation bleibe somit im Fokus der Sozialwirtschaft.

Auch Frank Buchheit und Stefanie Reiter vom AK Soziale Dienstleistungen in der DeGEval zogen ein positives Resumee zur Veranstaltung. Der mit rund 60 Teilnehmenden sehr gut besuchten Frühjahrstagung war es gelungen, im Handlungsfeld tätige Evaluierende sowie Programmverantwortliche aus dem deutschsprachigen Raum zusammenzuführen und dabei auch Personen zu erreichen, die bislang wenig Kontakte zur DeGEval hatten. An zwei Tagen fand ein intensiver interdisziplinärer Austausch statt, in welchem Erfahrungen geteilt und Möglichkeiten diskutiert wurden, wie mithilfe prozessgenerierter Daten in evaluativen Verfahren ein Mehrwert erzeugt und Wirkungen aufgespürt werden können. Hierbei wurden unterschiedliche Perspektiven, Ansätze und Methoden mit ihren Potenzialen und Grenzen beleuchtet. Es zeigte sich, dass nicht nur die Breite der vertretenen Disziplinen, Perspektiven und Ansätze, sondern auch die verwendeten Begrifflichkeiten (bspw. Evalua- 
tion, Monitoring $)^{8}$ Diskussionen anregen und Anschlussfragen aufwerfen. Die rege Beteiligung und Offenheit der Tagungsteilnehmenden führten somit zu einem spannenden Austausch mit wechselseitigen Anregungen. Es wurde von vielen Interesse artikuliert, die begonnenen feldübergreifenden Diskussionen weiterzuführen und zu vertiefen. Der AK Soziale Dienstleistungen wird sich dem Thema weiter widmen.

8 In diesem Kontext wurde auf das Glossar der Standards für Evaluation, abrufbar unter https:// www.degeval.org/degeval-standards/glossar-der-standards-fuer-evaluation/ [12.01.2020], verwiesen. 